\title{
Design of UWB pulse radio transceiver using statistical correlation technique in frequency domain
}

\author{
M. Anis and R. Tielert \\ Institute for Microelectronics, Technical University of Kaiserslautern, Germany
}

\begin{abstract}
In this paper, we propose a new technique to extract low power UWB pulse radio signals, near to noise level, using statistical correlation technique in frequency domain. The receiver consists of many narrow bandpass filters which extract energy either from transmitted UWB signal, interfering channels or noise. Transmitted UWB data can be eliminated by statistical correlation of multiple bandpass filter outputs. Super-regenerative oscillators, tuned within UWB spectrum, are designed as bandpass filters. Summers and comparators perform statistical correlation.
\end{abstract}

\section{Introduction}

In the last few years, UWB pulse radio has become attractive for low power and low cost short range applications, especially in wireless body area networks. WBAN requires innovative solutions to meet the energy consumption budget of the autonomous sensor nodes. Today's low power radio systems like Bluetooth and Zigbee do not meet these requirements, but the pulse based UWB reaches the target. It is an effective technique to deliver short range communication, using very short pulses and wide spectrum, enables coexistence with other narrow band radio systems. To mitigate the effects of interference to UWB from noise and other radio systems, we propose statistical correlation technique in frequency domain.

This paper describes the design of the first UWB CMOS transceiver, having ability to extract the UWB signal from the noise level. Our proposed architecture is based on simple on-off keying for low and medium data rate UWB system. The transmitter generates a pseudo random pattern of UWB impulses in a sub nanosecond time scale. The receiver consists of many narrow band pass super regenerative oscillators

Correspondence to: $\mathrm{M}$. Anis

(anis@eit.uni-kl.de)

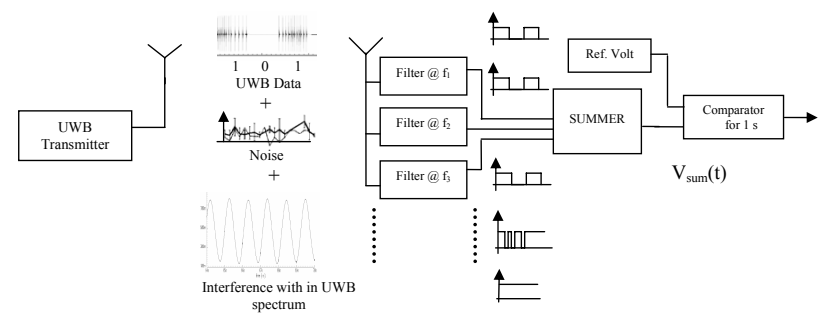

Fig. 1. OOK Low Data Rate UWB RF Front End Block Diagram.

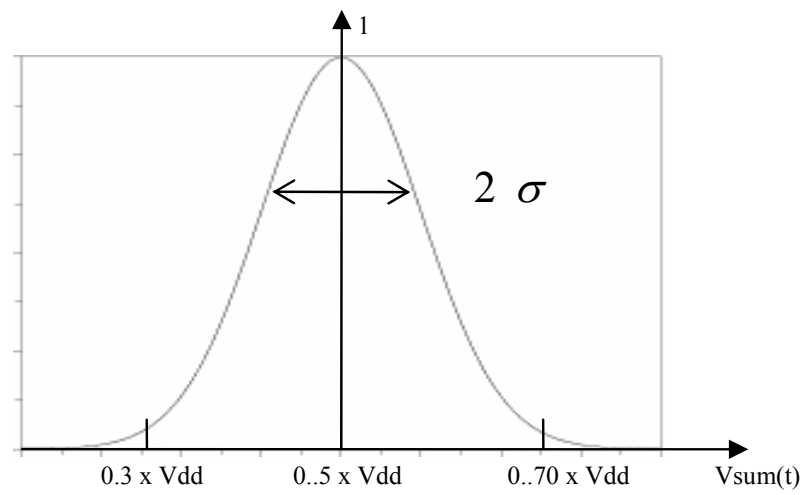

Fig. 2. The Probability distribution function of Vsum(t), in the absence of UWB data.

(SRO), tuned within UWB spectrum, acting as narrow bandpass filters. These SROs can be triggered either by the noise, interference or either by transmitted UWB data. Noise and interferences due to other radio channels are uncorrelated in all filter (SRO) outputs. UWB data are extracted after the statistical correlation of multiple bandpass filter outputs. Statistical correlation is performed by summer and comparator.

Published by Copernicus Publications on behalf of the URSI Landesausschuss in der Bundesrepublik Deutschland e.V. 


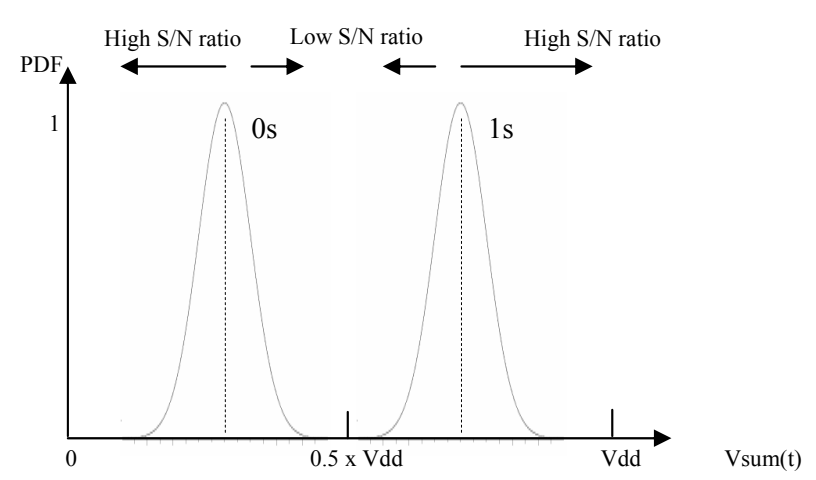

Fig. 3. Probability distribution function of Vsum(t), in the presence of UWB data.

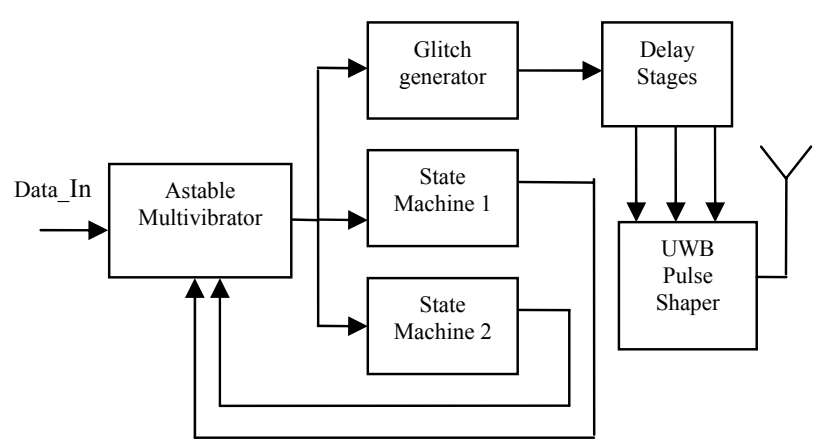

Fig. 4. Block diagram of UWB transmitter.

\section{System overveiw}

The proposed architecture based on statistical correlation in frequency domain is designed to extract the UWB signals which are embedded into noise and interfering channels. The UWB transmitter generates the pseudo random pattern of UWB impulses during on time of on-off modulation keying. The maximum energy level of UWB signal is near to noise level.

Figure 1 shows the UWB receiver which consists of multiple narrow band pass filters, tuned within the UWB spectrum. These filter banks will extract energy either from UWB transmitted data, noise or interfering channels. Outputs of filter banks due to noise are uncorrelated in each channel. Narrow band interfering channels like WLAN can disturb one or two filter channels. When the transmitted UWB data with embedded noise and interfering channels are present at antenna, a majority of the filter bank outputs will be correlated due to UWB data. Filter banks of frequency range $6 \mathrm{GHz}-$ $8.5 \mathrm{GHz}$ are designed by super-regenerative oscillators. The SRO was invented in 1922 by Armstong, well suited for short range low power applications. The SRO is turned on and off by a quench signal. The start up time of the SRO depends on the matching of tuned LC tank frequency and signal fre-

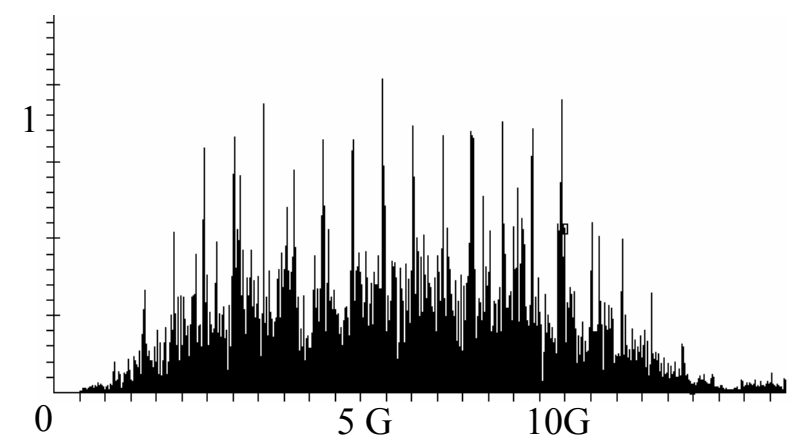

Fig. 5a. Normalize power density spectrum of periodic UWB impulses.

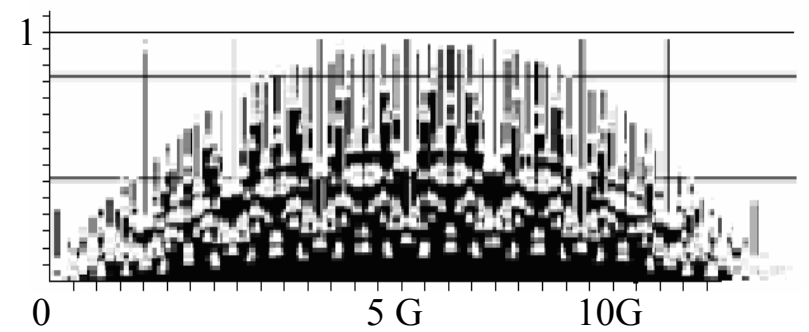

Fig. 5b. Normalize power density spectrum of PN sequence of UWB impulses

quency of received input. Twelve SROs, each using an LC filter tank have been integrated in our test structure. The output of every SRO is connected with envelop detectors, in order to get the output of each channel in digital form.

The most critical point is the biasing of SROs. The bias point of all SROs is adjusted so that average output voltage of each channel should be half of the supply voltage. In the absence of RF UWB signals, regulator increases the bias current of all SROs to make them sensitive to noise level, which means that every SRO has 50\% probability to be triggered due to noise and the summation of all filter (SROs + envelop det.) outputs Vsum(t) has a Gaussian distribution, as shown in Fig. 2. When the modulated UWB data having energy level near to noise is radiated by transmitter, the probability to detect $1 \mathrm{~s}$ increases in all channels. The regulator starts to decrease the bias current of all SROs, to maintain the average output voltage of each channel at the half of supply voltage. The UWB data can be extracted by comparing the summer output to the half of supply voltage.

$\begin{aligned} V_{\text {sum }}(t)>0.5 \times V d d & \Rightarrow 1 \\ V_{\text {sum }}(t)<0.5 \times V d d & \Rightarrow 0\end{aligned}$

Figure 3 shows the probability distribution function of Vsum $(t)$ in the presence of UWB data. The distribution functions for detecting $1 \mathrm{~s}$ and $0 \mathrm{~s}$ move towards the $50 \%$ of supply voltage for signals having small $\mathrm{S} / \mathrm{N}$ ratios and move 


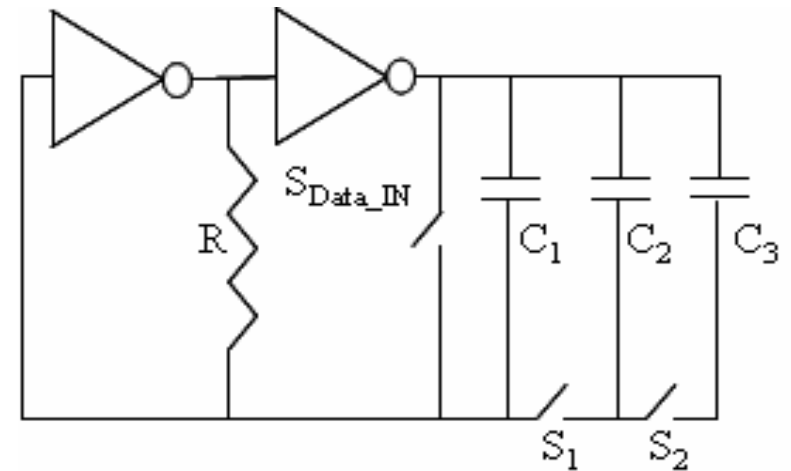

Fig. 6. Astable multivibrator with additional switches and capacitors.

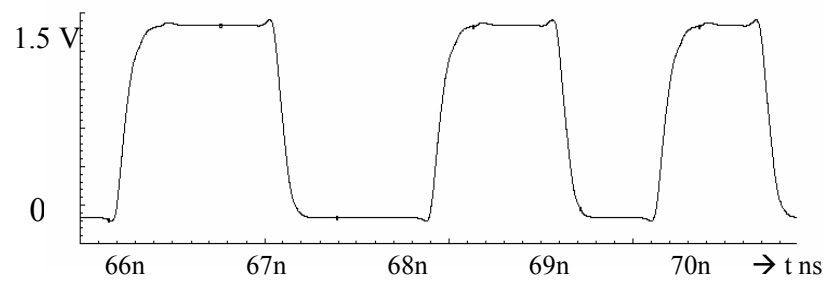

Fig. 7a.Pseudo random pattern at the output of astable multivibrator.

away from the $50 \%$ of supply voltage for signals having high $\mathrm{S} / \mathrm{N}$ ratios as shown in Fig. 3.

\section{UWB transmitter}

A fully integrated pulse generator for impulse based UWB system, covers the spectrum of $6-8.5 \mathrm{GHz}$, and was designed on $0.18 \mu \mathrm{m}$ CMOS technology. On/off modulation scheme is used. The basic structure consists of four parts, astable multivibrator with state machines, glitch generator, delay stages and UWB pulse shaper, shown in Fig. 4. Astable multivibrator (Baker et al., 1997) with state machines is used to generate the pseudo random (PN) pattern of UWB impulses. The periodic repetition of impulses generates the large spikes over the UWB spectrum. The PN sequence is used to flatten the UWB spectrum (Maggio et al., 2001). The simulation for power spectrum of periodic and $\mathrm{PN}$ sequence of impulses are shown in Figs. 5a, b.

The basic structure of astable multivibrator can generate periodic signals of pulse width $\mathrm{t} \sim 0.65 \mathrm{RC}$. Three switches $\left(\mathrm{S}_{\text {Data_In }}, \mathrm{S}_{1}, \mathrm{~S}_{2}\right)$ and two capacitors $\left(\mathrm{C}_{2}, \mathrm{C}_{3}\right)$ are added to the astable multivibrator as shown in Fig. 6. Switch $\mathrm{S}_{\text {Data_In }}$, connected to input digital data, will turn the multivibrator on and off. On-off keying modulation is used to transmit UWB pattern. When the switch is closed, multivibrator will be off and UWB pulse shaper will not transmit the UWB impulse. When the switch is open, multivibrator generates the pattern

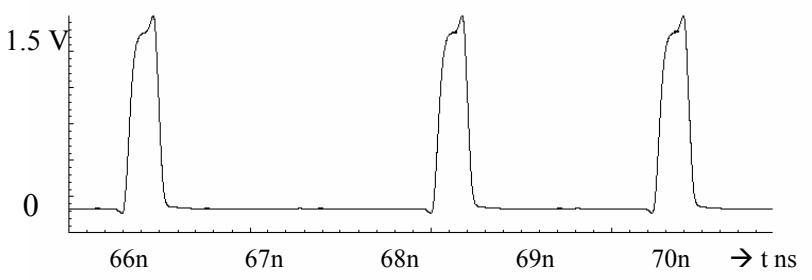

Fig. 7b. Pseudo random pattern at the output of glitch generator.

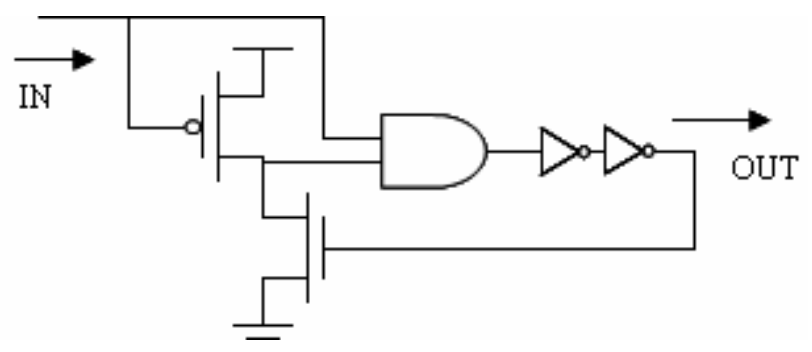

Fig. 8. Circuit diagram of basic glitch generator.

of pulse width $\mathrm{t} \sim 0.7 \mathrm{RC}_{e q}$, where $\mathrm{C}_{e q}$ is the sum of capacitances selected by state machines and parasitics of connected transistors. Initially the multivibrator generates a periodic rectangular pattern due to capacitor $\mathrm{C}_{2}$. The output of multivibrator is connected to the clock signal of two different state machines. These state machines generate the defined pattern used to select and deselect the capacitors by switches S1 and S2. Due to the changing of $\mathrm{C}_{e q}$ pulse width of the multivibrator changes, resulting in different clock rates of the cycles which generates the pseudo random pattern at the output, as shown in Fig. 7 a.

The UWB pulses require sharp rising and falling transitions. Due to the different rise and fall transition times of the multivibrator, two different pulse shapes and spectrum will be generated. In order to avoid this problem, very small glitches are generated on each rising edge of pattern. Figure $7 \mathrm{~b}$ shows the simulation results of the PN sequence at the output of the glitch generator. Figure 8 shows the circuit diagram of basic glitch generator. The delay stages are designed by a chain of basic CMOS inverters (Jeong et al., 2004). Delay stages generate three delayed small glitches. The delays are adjusted by the W/L ratios of inverters. These delays in between the glitches are smaller than the widths of glitches. These three glitch stages are connected to a UWB pulse shaper, consisting of three $\mathrm{LC}$ resonators tuned within 6-8.5 GHz range. Chirped UWB pulses with flat spectrum of $6-8.5 \mathrm{GHz}$ are achieved after mixing the outputs. Figure 9 shows the delay stages and UWB pulse shaper. Figure 10 shows the simulated pattern of UWB pulses at the load of $50 \Omega$. 


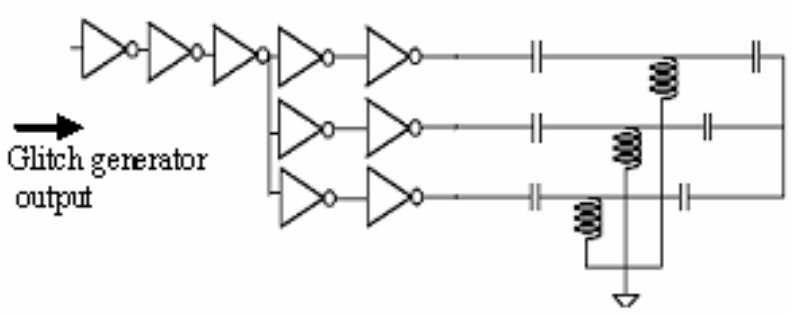

Fig. 9. Delay Stages and UWB Pulse Shaper.
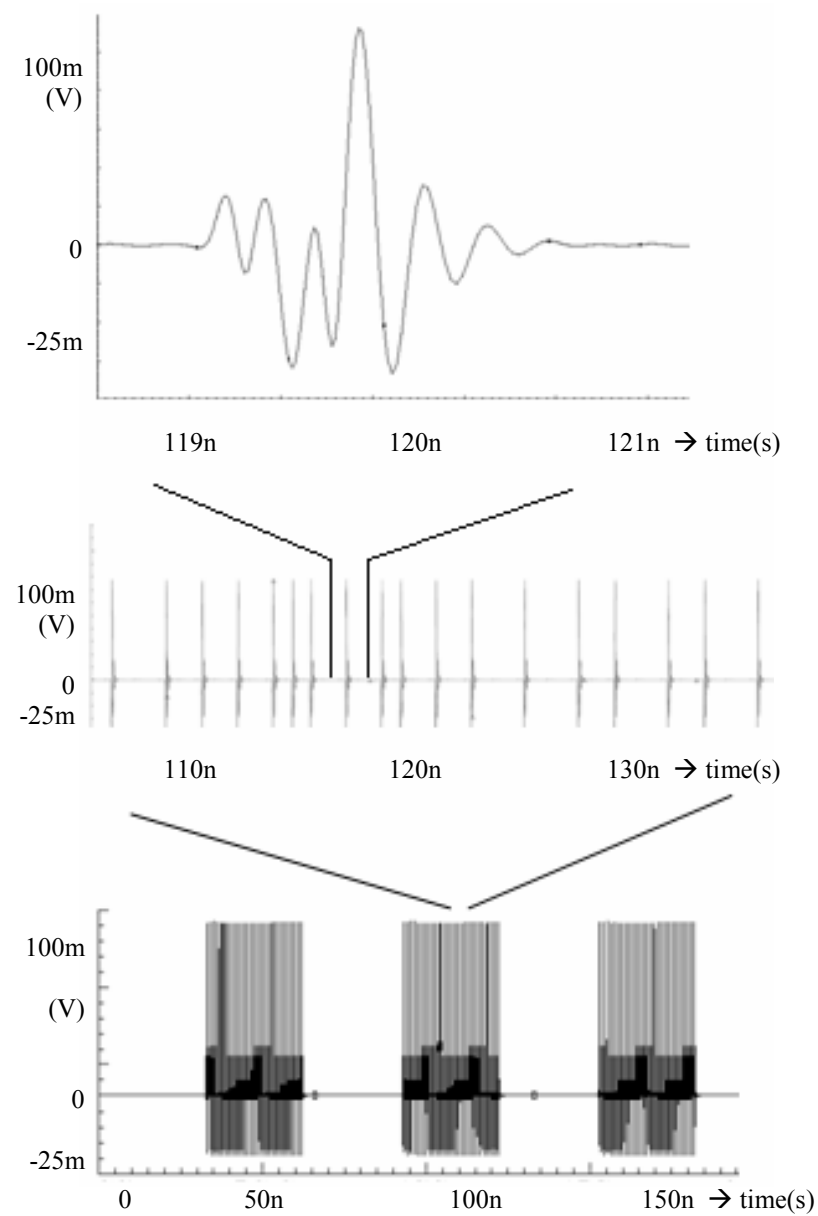

Fig. 10. The simulated pattern of UWB pulses at the load of $50 \Omega$.

\section{UWB receiver}

A fully integrated UWB pulse receiver covering the spectrum from $6-8.5 \mathrm{GHz}$ has been implemented in $0.18 \mu \mathrm{m}$ CMOS technology. Figure 1 shows the block diagram of the receiver. The simple low power system consists of isolation amplifier, super regenerative oscillators, envelop detectors, data synchronizers and regulated current control units, as shown in Fig. 11. The RF UWB signal is first fed to the isolation amplifier (IA). This common gate amplifier provides isolation of

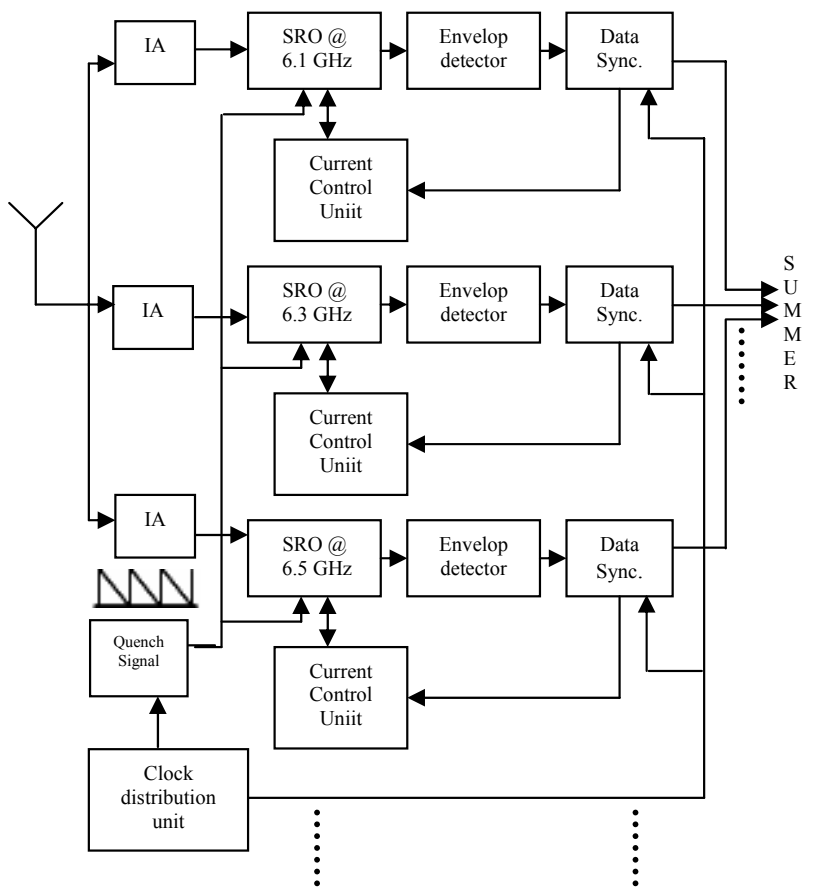

Fig. 11. Block diagram of multiple narrow band filter banks.

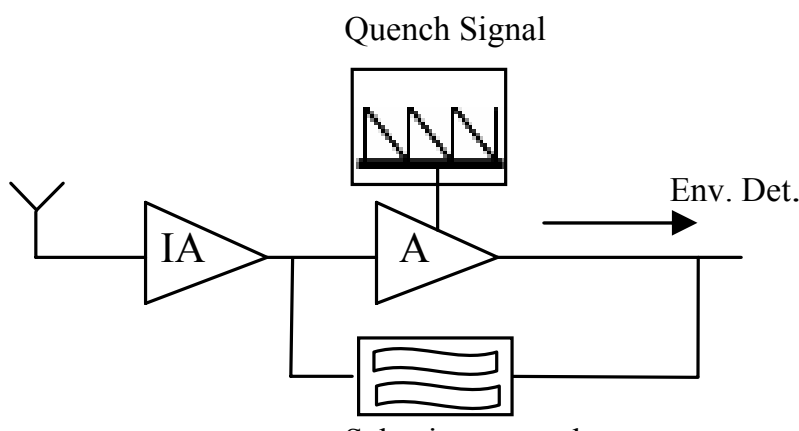

Selective network

Fig. 12. Block diagram of super-regenerative oscillator.

antenna and SROs. The signal then goes to SROs controlled by a feedback network as shown in Fig. 12. These oscillators are tuned within the range of the UWB signal spectrum 6$8.5 \mathrm{GHz}$. All oscillators are turned on and off by one quench generator. The start up time of the individual oscillators depends on the received input power and its frequency. The oscillator will start early, when the received signal is near to the center frequency of the oscillator. Without any RF signal, the start up time of oscillators depends on the feedback gain and thermal noise (Moncunill et al., 2005). Figure 13 shows the simulation results of four different SROs with on -off modulation for UWB. Selectivity and sensitivity, frequency and shape of the quench signal, minimum and maximum levels of bias currents of oscillators are critical issues, require 


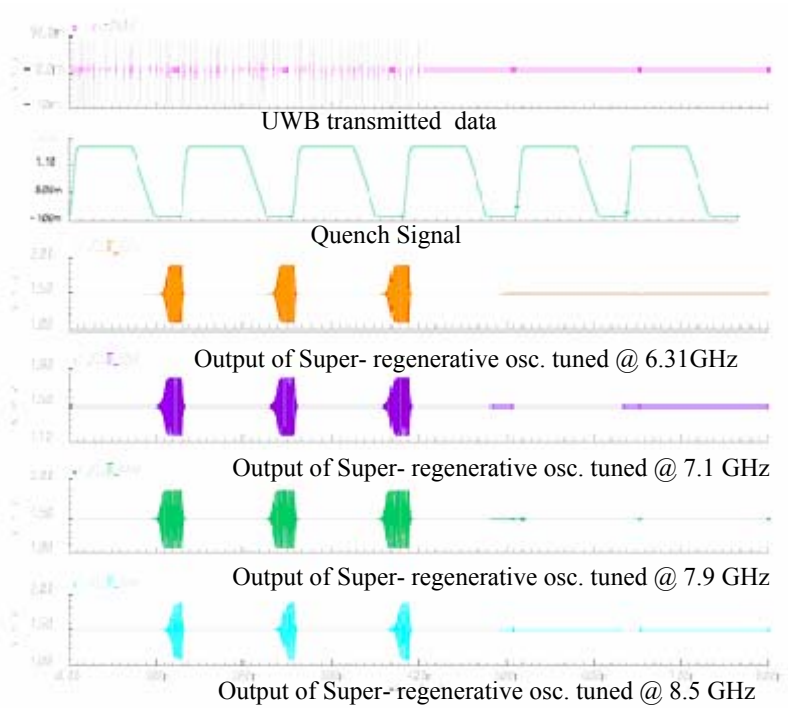

Fig. 13. The simulation results at the output of four SROs with UWB data.

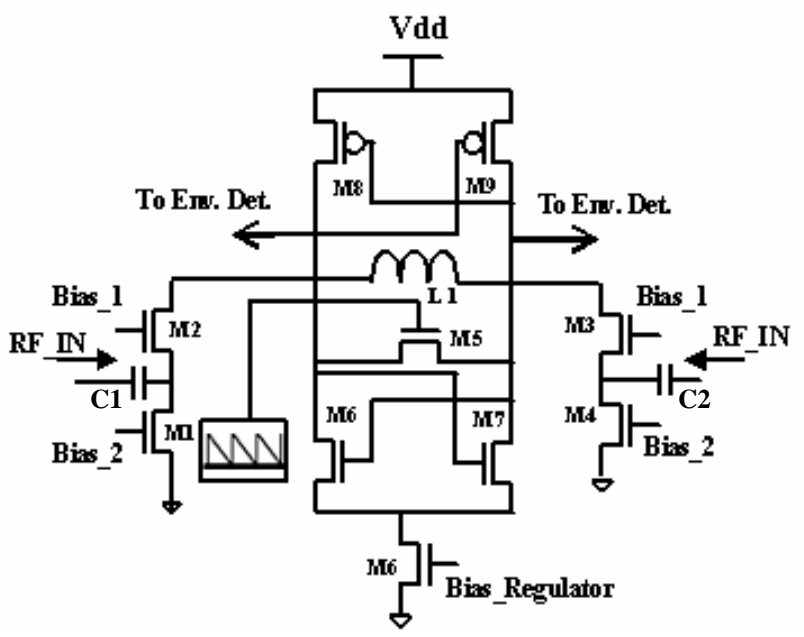

Fig. 14. The circuit diagram of isolation amplifier and super- regenerative oscillator.

detailed analysis as presented in (Favre et al., 1998; Vouilloz et al., 1998). The adjustment of bias points of all oscillators and the slope of the quench signal during start up of oscillators has to be optimized, in order to meet the bandwidth requirements of the filters.

The bias point of the individual channels is adjusted by the regulator so that all oscillators are sensitive to the noise level. Due to the biasing, there is $50 \%$ chance that oscillators will start to oscillate or remain off during the turn on time of the quench signal. An envelop detector senses the magnitude of the oscillations and provides the digital output. The transmitted UWB data is not synchronized with the quench signal. Data synchronizers as simple flip flops are used to

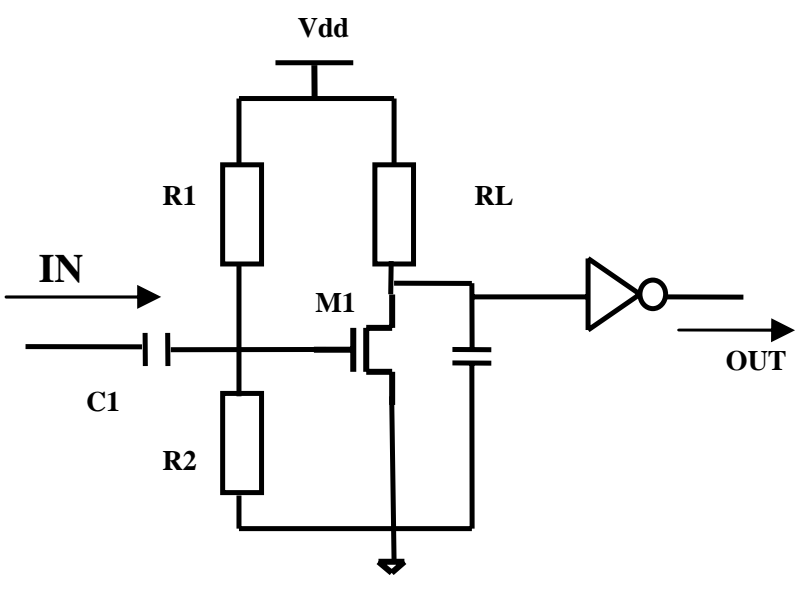

Fig. 15. The circuit diagram of envelop detector.

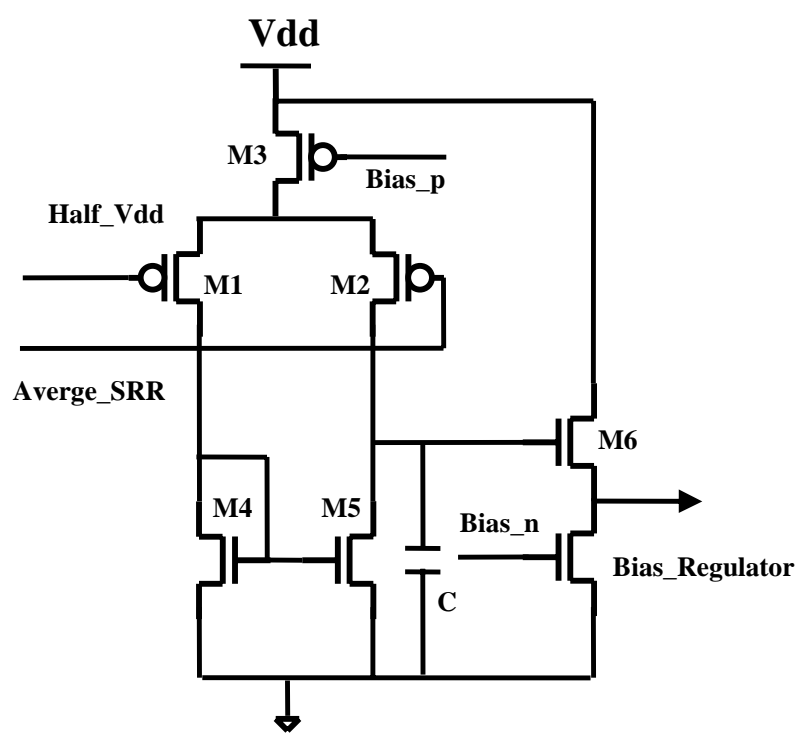

Fig. 16. The circuit diagram of current control unit.

synchronize the outputs of all envelop detectors with the reference clock, generated by the clock distribution unit. The average voltage at the output of data synchronizers should be half of the supply voltage. The current control unit regulates the bias currents of individual oscillators by comparing the average output voltage of data synchronizers with the half of supply voltage. In order to fulfill this condition, the input data coding of UWB modulation must contain equal number of ones and zeros, like in 8bit/10 bit coding.

The shape and frequency of quench signal has an effect on the performance of SROs. Detailed analysis of different types of quench signals is explained in (Joehl et al., 2001; Vouilloz et al., 2001). According to the nyquist criteria, the quench frequency must be two times or more than the transmitted UWB data rate. The quench signal is derived from 


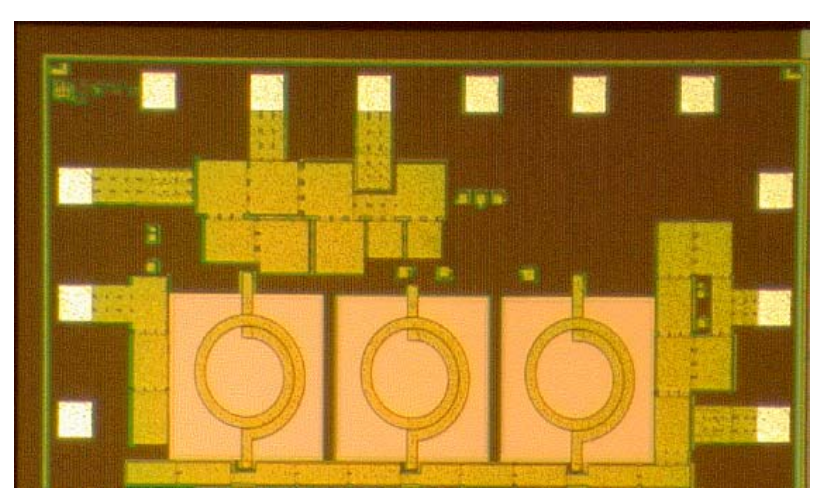

Fig. 17. Die photograph.

the reference clock, having a fast low to high transition and a slow high to low transition. When the slope of the quench signal is low, SROs are sensitive in narrow frequency bands, consuming low power and allowing low data rate. When transition is fast during starting of oscillators, SROs will be sensitive in a wide frequency band, consuming high power and allowing high data rate (Moncunill et al., 2005; Feick and Rojas, 1997). The statistical correlation between multiple channels requires a low bandwidth of the SROs in order to separate the frequency channels. The center frequencies and bandwidth of all SROs are designed in a way that cross coupling between adjacent channels is negligible. In the designed test chip, SROs of adjacent frequency bands are placed most far from each other to reduce the effect of coupling, as illustrated in Fig. 19

The schematic of SRO is shown in Fig. 14. The RF signal of the UWB antenna goes to the source of transistors M2 and M3, which are common gate isolation amplifiers. M1 and M4 provide the bias currents for this IA. The outputs of IA are directly connected to the differential LC oscillator. Twelve integrated inductors are used to cover the $6-8.5 \mathrm{GHz}$ range. The quench signal is connected to the gate of transistor M5, which turns the oscillator on and off. Transistor M6 provides the regulated bias current of the oscillator. Fig. 15 shows the circuit diagram of an envelop detector. Capacitor $\mathrm{C} 1$ is used to remove the DC component from the oscillation. Resistors R1 and R2 are adjusted to keep the transistor M1 at its threshold voltage, hence transistor M1 acts as RF rectifier. Figure 16 shows the circuit diagram of a current control unit. The circuit is comparing the average output voltage of data synchronizers with half of Vdd. Capacitor $\mathrm{C}$ is used to integrate the DC-free modulation.

The output of data synchronizers from all channels are added by the summer circuit. The summer output is connected to a clocked comparator. The reference voltage of the comparator is adjustable between 0 to Vdd, used to identify ones and zeros of transmitted data. If the UWB transmitter is off, the bias current of SROs will start to increase by regulator to make them sensitive to noise level, which means
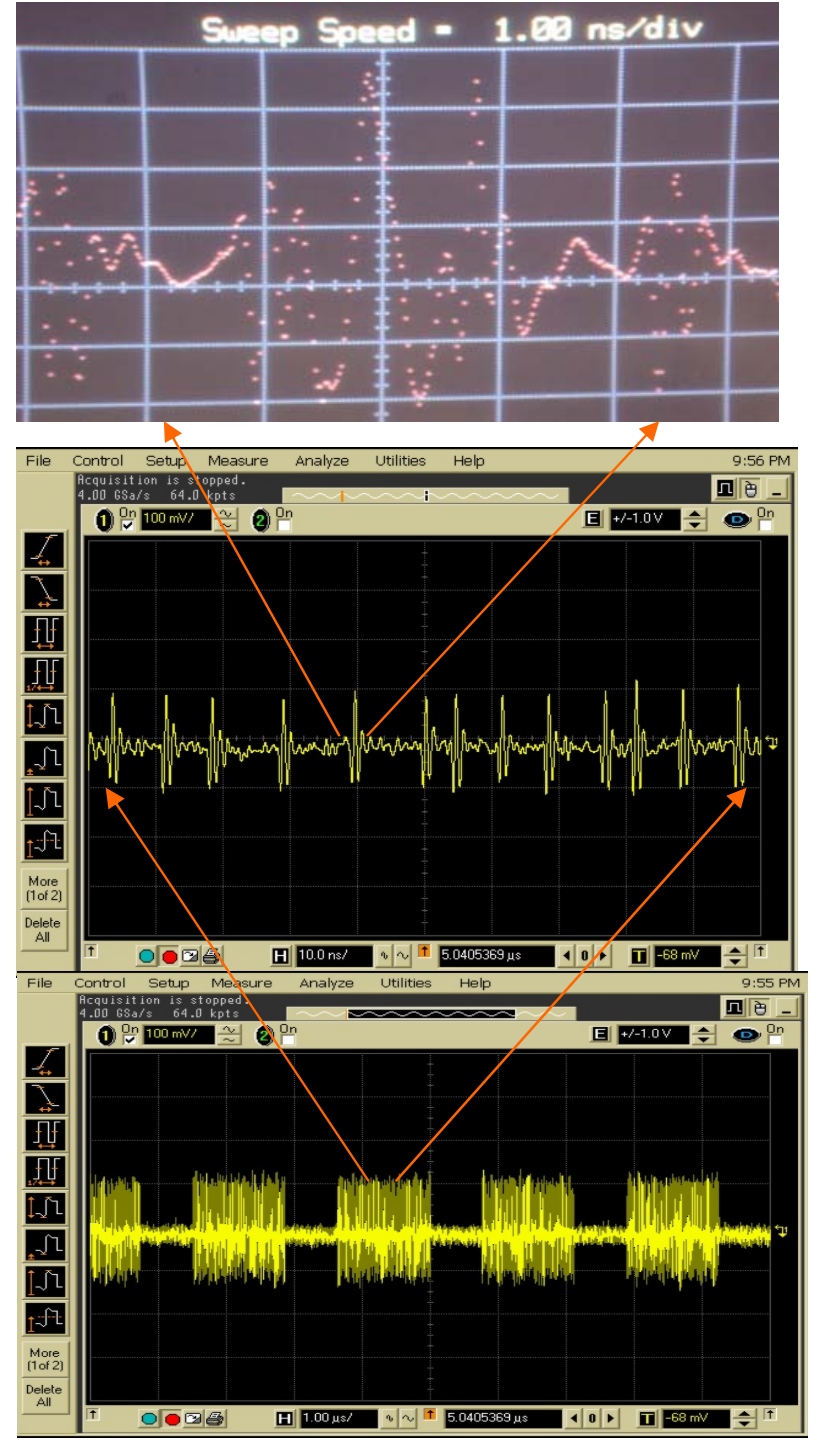

Fig. 18. The measurement results for UWB transmitter at the load of $50 \Omega$.

that the triggering of all channels is only due to noise, and summer output has Gaussian distribution as shown in Fig. 2. When the UWB transmitter radiates pseudo random pattern of pulse during on - off keying, the bias current of all SROs starts to decrease to make the all channels less sensitive, allowing for an improved $\mathrm{S} / \mathrm{N}$ ratio. The maximum number of continuous ones and zeros in modulated UWB data is limited due to the RC time constant of regulator loop. 


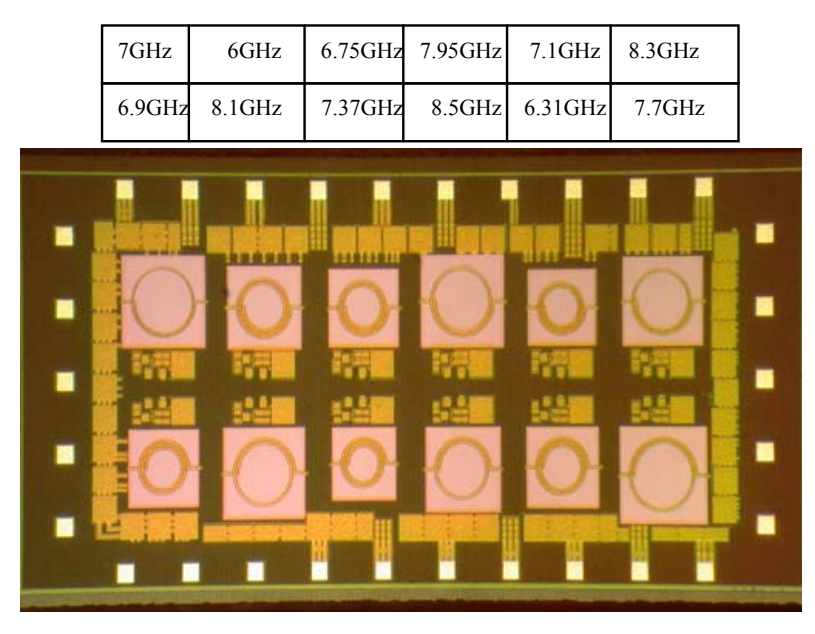

Fig. 19. Die photograph for UWB receiver.

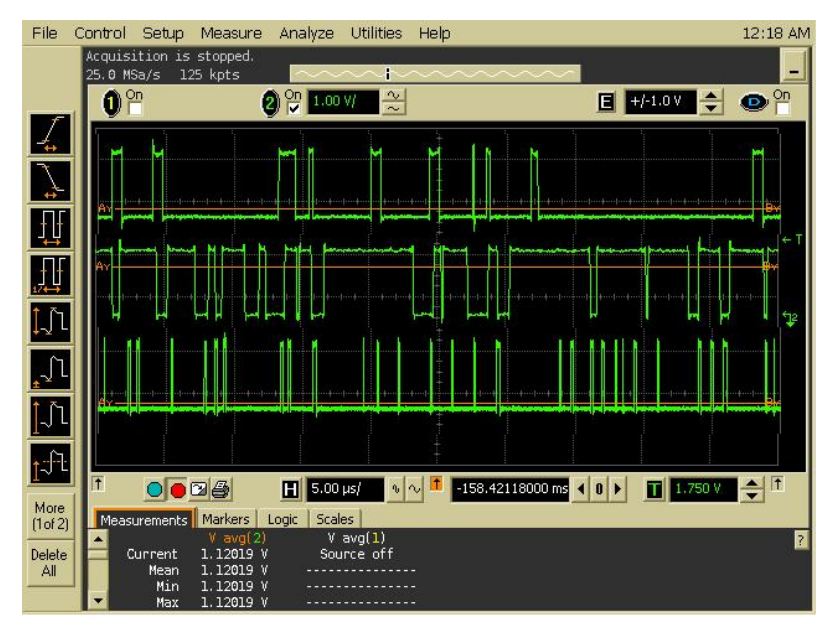

Fig. 20. The uncorrelated outputs of three adjacent channels.

\section{Experimental results}

\subsection{UWB transmitter}

The UWB $(6 \mathrm{GHz}-8.5 \mathrm{GHz})$ transmitter has been implemented on $0.18 \mu \mathrm{m}$ CMOS technology, active area of $0.65 \mathrm{~mm}^{2}$. The power consumption is $0.6 \mathrm{~m} \mathrm{~W}$ for transmitting UWB pulses with on -off modulation keying at the data rate of $4 \mathrm{MHz}$. The die photo is shown in Fig. 17. The measurement of UWB transmitter, shown in Fig. 18, can be compared with the simulation results shown in Fig. 10

\subsection{UWB receiver}

The test structure has been implemented on $0.18 \mu \mathrm{m}$ CMOS technology, active area of $2.5 \mathrm{~mm}^{2}$. The UWB receiver, designed for maximum quench rate of $8 \mathrm{MHz}$, can receive maximum data rate of $4 \mathrm{MHz}$ with power consumption of $2 \mathrm{~mW}$

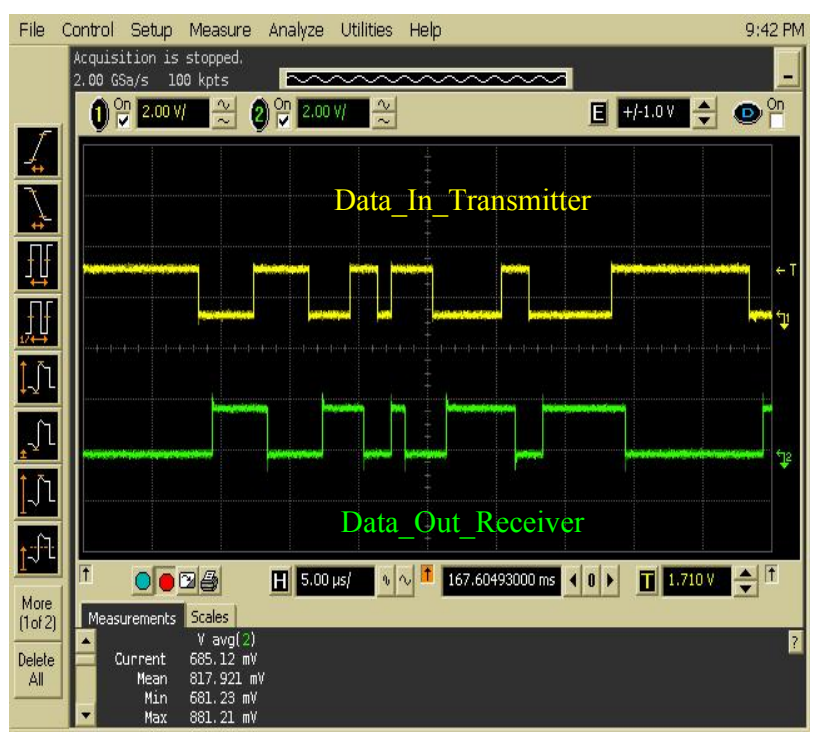

Fig. 21. The measured output at the receiver for radiated pattern from transmitter (data out is delayed and inverted).

from $1.5 \mathrm{~V}$ supply. The die photograph is shown in Fig. 19. The adjacent frequency channels are placed far from each other to reduce the effect of coupling. The outputs of three adjacent channels, shown in Fig. 20, are uncorrelated to each other in the absence of UWB data, triggering only due to noise. Figure 21 shows the input data pattern to the transmitter. The transmitter generates pseudo random pattern of UWB pulses during low input level and off during is high input level. The peak voltage of these pulses is around $20 \mathrm{mV}$. The receiver input has been coupled to the UWB transmitter by a $60 \mathrm{~dB}$ attenuator. The output of UWB receiver after statistical correlation of all channels is shown in Fig. 21.

\section{Conclusions}

The new architecture to receive the low power UWB pulse radio signals by statistical correlation of multiple channels has been proposed in this paper. The transmitter radiates pseudo random pattern of UWB pulse in the spectrum from 6-8.5 GHz. The receiver extracts the UWB pulses of energy level near to noise. Narrow band interfering transmitters are detected and eliminated by statistical correlation of twelve narrow bandpass SROs.

\section{References}

Baker,R. J., Li,H. W., and Boyce, D. E.: CMOS Circuit design, Layout, and Simulations, IEEE press series on Icroelectronics System, 1997.

Favre, P., Joehl, N., Vouilloz, A., Deval, P., Dehollain, C., and Declerq, M.: A 2-V 600 uA 1GHz BiCMOS super-regenerative re- 
ceiver for ISM applications, IEEE J. Solid- State Circuits, 33(12), 2186-2196, 1998.

Feick, R., Rojas, O., and Mackay, A.: Modeling and simulation of the super regenerative receiver, IEEE Trans. On Consumer Electronics, 43(2), 92-102, 1997.

Jeong, Y., Jung, S., and Liu, J.: A CMOS impulse generator for UWB Wireless communication systems", in proc. ISCAS, 1291322004.

Joehl, N., Dehollain, C., Favre, P., Deval, P., and Declercq, M.: A low power $1-\mathrm{GHz}$ super-regenerative transceiver with timeshared PLL control, IEEE J. Solid State Circuit, 36(7), 10251031, 2001.

Moncunill-Geniz, F. X., P-Schönwälder, P., and del Aguila- Lopez, F.: New superregenerative architectures for direct sequence spread spectrum communications, IEEE Trans. on Circuits and Systems-II Express Briefs, 52(7), 415-419, 2005.
Moncunill-Geniz, F. X.,P-Schönwälder, P., and M-Casals, O.: A generic Approach to the theory of Super regenerative reception, IEEE Tran. on Circuits and Systems-I: Regular Paper, 52(1), 5470, 2005.

Maggio, G. M., Rulkov, N., and Reggiani, L.: Pseudo- chaotic time hopping for UWB impulse radio, IEEE Trans. On circuit and system-I, 48(12), 1025-1031, 2001.

Vouilloz, A., Declercq, M., and Dehllain, C.: A low power CMOS Super- Regenerative Receiver, IEEE J. Solid State Circuit, 36(3), 440-451, 2001.

Vouilloz, A., Declerq, M., and Dehollain, C.: Selectivity and sensitivity performance of super-regenerative receivers, in proc. ISCAS', 325-328 98. 